\title{
Analysis of Strain Localization at High Angle Grain Boundaries During Creep of a Polycrystalline Superalloy Using SEM-Based Digital Image Correlation
}

\author{
C.E. Slone ${ }^{1}$, M.J. Mills ${ }^{1}$ \\ 1. Center for Electron Microscopy and Analysis, The Ohio State University, Columbus, OH
}

High-temperature deformation of superalloys is a complicated process involving many possible mechanisms that depend on temperature, applied load, and microstructure [1]. Observation and quantification of microstructural features are critical to understanding and predicting macroscopic behavior. Notably, there is a growing emphasis on coupling multi-scale material characterization to local mechanical behavior; this type of work is essential for understanding the deformation of materials exhibiting complex, heterogeneous microstructures [2].

In this work, polycrystalline Ni-based superalloy IN740H was deformed to small strains $(\sim 0.1 \%)$ at $750^{\circ} \mathrm{C}$ under constant loads ranging from $220-330 \mathrm{MPa}$. Alloy $740 \mathrm{H}$ has previously demonstrated creep behavior governed by microstructural heterogeneities with premature failure observed along regions of alternating precipitate coarsening and depletion [3]. Prior to deformation in this work, selected regions of interest were scanned using electron backscatter diffraction (EBSD) to determine grain orientations and boundary types. $\mathrm{HfO}_{2}$ patterns were applied to the same regions of interest via an e-beam lithography process to serve as a pattern for strain mapping using digital image correlation (DIC). These patterns contained both a pseudo-random speckle pattern (speckle edge length 200nm, average spacing 200nm) and grid lines for measurement of grain boundary sliding; see Figure 1(a). Scanning electron microscope (SEM) images of the $\mathrm{HfO}_{2}$ patterns were acquired before and after deformation and correlated using VIC2D software from Correlated Solutions. This procedure produced full-field strain maps (spatial resolution $\sim 1 \mu \mathrm{m}$ ) that could be subsequently matched with previous EBSD scans to directly examine the relationship between microstructure and mechanical behavior.

Carter et al. [4] previously demonstrated a similar technique for polycrystalline superalloys featuring in situ SEM-DIC. This work utilized a heated SEM tensile stage to observe deformation at high temperatures and large loads that produced extensive deformation (>10\%) over several hours. In situ SEM-DIC has also been explored by Kammers et al. [5], who presented a systematic analysis of distortions and artifacts incurred during the imaging process. Most recently, Stinville et al. [6] showed the viability of these techniques at sufficiently small length scales to capture localized deformation around individual slip traces. The present work advances this science by applying SEM-DIC as an ex situ technique: this enables prolonged high temperature testing (at strain rates relevant to service conditions) while preserving the ability to create full-field strain maps with spatial resolutions smaller than $1 \mu \mathrm{m}$. The present work has carefully quantified the errors introduced by an ex situ method and a representative error map is shown in Figure 1(b).

A typical strain map from a sample deformed in creep is shown in Figure 1(c); the strain map has been overlaid with grain boundaries from an EBSD scan of the same region prior to deformation. These results indicate significant strain localization associated with specific microstructural features; it can be shown that these are high angle grain boundaries (HAGBs), whereas boundaries without localization are $\Sigma 3$ twin boundaries. Strain measurements at the high angle boundaries are up to two orders of magnitude larger than background measurements ( $2 \%$ vs $0.02 \%)$. Given the underlying orientation data from EBSD scans, 
it is also possible to compare regions of strain localization to the Schmid factor of each grain. This comparison shows that there is no strong correlation between the measurements; however, analysis of macroscopic test data indicates a creep stress exponent of $n \approx 1.1$. Stress exponent values in this range typically suggest a diffusional mechanism for creep (accommodated by some motion or sliding at grain boundaries), which is supported by the lack of correlation between DIC results and Schmid factor calculations (since the latter apply to cases of slip via dislocation motion rather than diffusion). A diffusional mechanism is also supported by the disparity in response between HAGBs and twin boundaries since the diffusion coefficient is much smaller for the latter. If confirmed, this represents an excellent opportunity to quantitatively study the manner in which diffusional creep is accommodated in polycrystalline superalloys.

In addition to SEM-DIC, this work also includes the use of other techniques to yield microstructural information. Scanning transmission electron microscopy (STEM) analysis is critical to imaging and quantifying crystal defects such as dislocations and microtwins; electron channeling contrast imaging (ECCI) is also used to quickly examine defect content over statistically significant areas. The results described above will be discussed in context as the intersection of traditional characterization techniques (EBSD, EDS, STEM) with emerging experimental methods (DIC).

\section{References:}

[1] Unocic et al. Materials Science and Engineering A. 483-484 (2008) 25-32.

[2] Walley et al. Experimental Mechanics. 52 (2012) 405-416.

[3] Bechetti et al. Metallurgical and Materials Transactions A. 46 (2014) 739-755.

[4] Carter et al. Materials Science \& Engineering A. 605 (2014) 127-136.

[5] Kammers et al. Experimental Mechanics. 53 (2013) 1743-1761.

[6] Stinville et al. Acta Materialia. 98 (2015) 29-42.

[7] This material is based upon work supported by the National Science Foundation Graduate Research Fellowship Program under Grant No. DGE-1343012. Any opinions, findings, and conclusions or recommendations expressed in this material are those of the authors and do not necessarily reflect the views of the National Science Foundation.
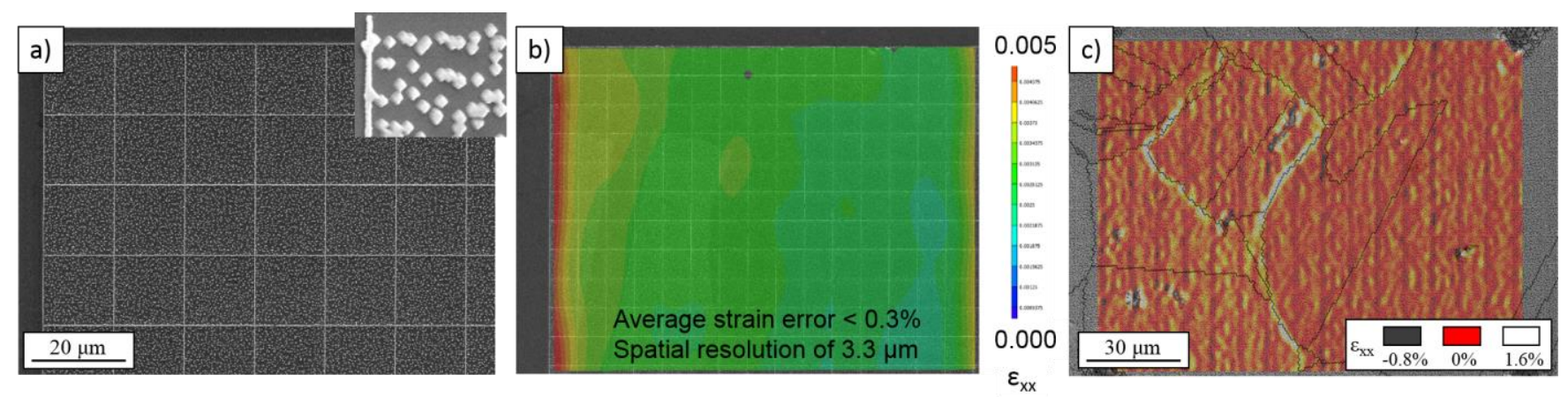

Figure 1: a) $\mathrm{HfO}_{2}$ speckle and grid patterns (inset: magnified speckle pattern), and b) correlation on an undeformed sample; any measured strain on this pattern is due to error in the ex situ process or strain calculation. c) Strain map in IN740H produced by digital image correlation after $0.1 \%$ strain at $750^{\circ} \mathrm{C} /$ $232 \mathrm{MPa}$. Load was applied along the horizontal axis of the image. 